\title{
Criminologie
}

\section{Expérience communautaire pour les hommes pédophiles}

\section{Marc Daigle}

Volume 30, numéro 2, automne 1997

Violences familiales

URI : https://id.erudit.org/iderudit/017407ar

DOI : https://doi.org/10.7202/017407ar

Aller au sommaire du numéro

Éditeur(s)

Les Presses de l'Université de Montréal

ISSN

0316-0041 (imprimé)

1492-1367 (numérique)

Découvrir la revue

Citer cet article

Daigle, M. (1997). Expérience communautaire pour les hommes pédophiles. Criminologie, 30(2), 109-127. https://doi.org/10.7202/017407ar

\section{Résumé de l'article}

The evaluation of the self-help program "Le groupe d'entraide Amorce" for pedophilic men showed they were progressing regarding certain pedophilic characteristics, particularly with respect to a better integration of their adult sexuality. However, nobody in this program claimed to be "treating" pedophilia directly. In fact, all of them relied upon the sharing and supporting dynamics of a self-help group to face other related problems and to reach the preliminary but necessary steps for deeper changes. The beneficial effect of the program was felt regarding mainly stopping withdrawal, changing attitudes, increasing empathy, motivating for change, improving communication skills, discharging emotions, clarifying immediate problems, repositionning as adults, supporting when depressed or decreasing anxiety. The triangulation of the points of view of facilitators, external parties, and participants did confirm these results both regarding pedophilia and other problems. Beyond these effects, the evaluation showed that the main strengths of the program were: the support and sharing of experiences between participants ; the motivation and availability of those involved ; the openness to external critics, novelty, uniqueness, pro-gressiveness, communal, non-medical, and preventive approach of pedophilia ; and the respect for and "responsibilisation" of the participants. Some aspects of the program also needed to be improved and specific recommendations were provided to the administrators.
Ce document est protégé par la loi sur le droit d'auteur. L'utilisation des services d'Érudit (y compris la reproduction) est assujettie à sa politique d'utilisation que vous pouvez consulter en ligne.

https://apropos.erudit.org/fr/usagers/politique-dutilisation/ 
The evaluation of the self-help program "Le groupe d'entraide Amorce" for pedophilic men showed they were progressing regarding certain pedophilic characteristics, particularly with respect to a better integration of their adult sexuality. However, nobody in this program claimed to be "treating" pedophilia directly. In fact, all of them relied upon the sharing and supporting dynamics of a self-help group to face other related problems and to reach the preliminary but necessary steps for deeper changes. The beneficial effect of the program was felt regarding mainly stopping withdrawal, changing attitudes, increasing empathy, motivating for change, improving communication skills, discharging emotions, clarifying immediate problems, repositionning as adults, supporting when depressed or decreasing anxiety. The triangulation of the points of view of facilitators, external parties, and participants did confirm these results both regarding pedophilia and other problems. Beyond these effects, the evaluation showed that the main strengths of the program were: the support and sharing of experiences between participants; the motivation and availability of those involved ; the openness to external critics, novelty, uniqueness, progressiveness, communal, non-medical, and preventive approach of pedophilia; and the respect for and "responsibilisation" of the participants. Some aspects of the program also needed to be improved and specific recommendations were provided to the administrators.

"Le groupe n'a pas réglé mon problème de pédophilie. Il m'a plutôt aidé à placer les choses... quand j'étais mêlé."

Témoignage d'un participant au groupe d'entraide Amorce

L'argumentation en faveur de l'utilisation des groupes d'entraide pour les « victimes » de l'inceste, sinon de la pédophilie, a déjà été exposée par

1. Ph.D., professeur au Département de psychologie, Université du Québec à TroisRivières, Case postale 500, Trois-Rivières, Québec, G9A 5H7. 
Herman (1981). Quant à l'efficacité générale des groupes d'entraide, elle semblerait par ailleurs bien démontrée, du moins dans certains domaines particuliers ou pour ce qui est de sa complémentarité avec le système de santé (Lavoie, 1989 ; Hedrick et al., 1992). Selon la définition retenue par Romeder (1989), les groupes d'entraide seraient :

Des petits groupes autonomes et ouverts qui se réunissent régulièrement. Victimes (sic) d'une crise ou d'un bouleversement commun dans leur existence, les membres de ces groupes partagent un vécu commun de souffrance et un sentiment d'égalité. Leur activité primordiale est l'entraide personnelle qui prend souvent forme de soutien moral, par le partage d'expériences et d'information, et par la discussion (p. 34).

En principe, cette formule devrait notamment aider les participants à reconnaître le pouvoir de changement qui leur appartient (Silverman, 1992).

Les fondateurs du groupe d'entraide Amorce, groupe communautaire créé pour les hommes pédophiles, n' avaient certes pas comme objectif d'encourager ceux-ci à se considérer comme des « victimes " (quoique la majorité d'entre eux aient déjà été « victimisés » dans leur enfance). Au contraire, ils voulaient plutôt les aider à se responsabiliser davantage. Cela étant bien clair dans leur esprit, ils entendaient toutefois leur permettre de partager leur « souffrance » commune afin de pouvoir aller plus loin dans leur cheminement. Une souffrance qui, vue de l'extérieur, s'appellerait errance sociale, désarroi, perte des acquis, isolement... puis, éventuellement, récidive.

Car une vérité fondamentale demeure : hors des institutions, peu d'aide est apportée aux hommes pédophiles, eux qu'on définit pourtant comme très vulnérables, même après un traitement. En effet, la « prise en charge thérapeutique » de ces agresseurs sexuels se fait surtout dans des établissements carcéraux ou dans des hôpitaux spécialisés après l'étape obligée de la condamnation. Ainsi, une partie des agresseurs sexuels condamnés, qu'ils soient pédophiles ou non, est soumise à des traitements institutionnels spécialisés, le plus souvent d'ơrientatión cognitive-comportementale. Or, même dans le cas de ces personnes, il n'est pas certain que l'efficacité du traitement soit toujours très grande, surtout si l'on utilise le critère de la non récidive (Pellerin et al., 1996). De plus, ces programmes institutionnels s'avèrent nécessairement très coûteux, notamment en comparaison de ce qui devrait aussi être offert aux autres délinquants (Vérificateur général du Canada, 1996).

Mais, par-delà ces débats qui, faut-il l'espérer, ne ralentiront pas le cheminement de la science dans ce domaine bien particulier, demeure toujours le problème des hommes pédophiles qui sont laissés à eux-mêmes dans la communauté, que ce soit avant ou après l'incarcération ou le traitement. 
Là-dessus, le même rapport du Vérificateur général relevait justement le manque de continuité entre les programmes institutionnels et communautaires, et ce, pour des personnes qui auraient pourtant besoin « d'un encadrement tout au long de leur vie " (Wilson, 1996, p. 29). D'où la nécessité de mettre en œuvre de nouveaux programmes dans la communauté, en s'inspirant notamment de la formule des groupes d'entraide. C'est ce qui a été réalisé dans la région de Montréal avec le groupe Amorce, dont l'évaluation est présentée dans cet article.

\section{DESCRIPTION DU PROGRAMME}

Le groupe Amorce a été mis sur pied par l'organisme L'entraide pour hommes de Montréal, lui-même actif depuis 1989. La démarche s'appuyait sur une demande du Service de probation du Québec en octobre 1992, laquelle résultait d'un cheminement amorcé dès 1989 avec, entre autres, un des hommes pédophiles qui participe encore au groupe. L'objectif était alors la création d'un « service d'entraide à l'intention des hommes pédophiles » dans le but de briser leur isolement. Il s'agissait donc d'entreprendre avec cette clientèle quelque chose qui soit différent de ce qui existait déjà au niveau thérapeutique. À la suite d'une consultation dans le milieu communautaire et thérapeutique, le programme a été développé par les animateurs de L'entraide pour hommes de Montréal. Il a finalement été subventionné par le ministère de la Santé et des Services sociaux, celui de la Sécurité publique du Québec, et par le Service correctionnel du Canada.

Au point de départ, le programme voulait aider les hommes pédophiles « à se responsabiliser, à briser leur isolement, à s'insérer dans un réseau d'entraide et à s' autocontrôler ». Plus spécifiquement, ce programme innovateur ${ }^{2}$ devait « leur donner la parole, favoriser le partage et le respect réciproque, amorcer un cheminement d'acceptation de leurs différences mais aussi un cheminement de croissance personnelle " (documentation de l'organisme). Dans l'ensemble, c'étaient donc là des objectifs bien ambitieux mais que les trois animateurs (non pédophiles) des rencontres acceptaient avec humilité. Ceux-ci possédaient déjà une solide expérience de l'entraide mais avec d'autres types d'hommes en difficulté. Par contre, ils pouvaient compter sur les conseils d'un « comité aviseur » de professionnels des milieux thérapeutiques et légaux.

2. À notre connaissance, seul le programme Phoenix utiliserait la formule de l'entraide avec des délinquants sexuels. Il est toutefois localisé à l'intérieur d'un pénitencier canadien, dans l'ouest du pays, et il fonctionne donc dans un contexte tout à fait différent. 
Les activités du groupe Amorce sont essentiellement des rencontres de discussions non dirigées qui ont lieu un soir par semaine. Les trois animateurs y réunissent une quinzaine de participants déjà accueillis individuellement au préalable. L'entrée au programme est continue, sans attente, et les participants peuvent donc en être à différentes étape de leur cheminement (contrairement à ce qui existe souvent en thérapie). Les animateurs se perçoivent justement comme des animateurs et non pas comme des thérapeutes, d'autant plus qu'ils n'ont pas une formation très spécifique en délinquance sexuelle. Néanmoins, ils maîtrisent des techniques d'intervention qui pourraient être qualifiées d'humanistes, non directives, un peu à la manière de Giarretto et Einfeld-Giarretto (1982, 1990), deux « thérapeutes » américains de la délinquance sexuelle intrafamiliale. Dans le contexte particulier d'un groupe d'entraide, ils se veulent donc des animateurs qui doivent encadrer et stimuler une dynamique qui, idéalement, appartient aux participants euxmêmes. Ces participants sont alors à la fois les objets « et » les outils de changement, ce qui complexifie évidemment la tâche d'évaluation, par opposition à un programme qui identifierait clairement clients et thérapeutes (Voir Kramer et Nash, 1992, sur cette difficulté).

Par ailleurs, la démarche d'évaluation du programme ne visait pas à démontrer l'efficacité relative de cette approche non directive parmi les différents types d'interventions offerts aux délinquants sexuels, d'autant plus qu'il ne s'agit justement pas ici d'intervention à proprement parler. Une méthodologie plus formative que sommative devait alors être adoptée. Il s'agissait donc de collaborer au développement du programme, tout en permettant de refléter fidèlement ce qui avait été fait jusqu'à présent. C'est pourquoi l'accent a été mis sur une évaluation du « processus » de changement lui-même, sans toutefois négliger certaines mesures de «l'atteinte des objectifs » spécifiques du programme.

\section{DEVIS D'ÉVALUATION}

L'évaluation du programme Amorce a été entreprise à la suite d'une démarche de réflexion des animateurs et des responsables du programme ${ }^{3}$. D'un autre côté, les organismes subventionnaires, après bientôt deux ans de fonctionnement du programme, désiraient aussi faire le point sur la situation. Ces derniers représentent donc ici un deuxième point de vue, soit celui de la société, tout comme les agents des organismes externes qui dirigent leurs clients vers ce programme. Plus loin dans la démarche d'évaluation,

3. L'exercice d'évaluation a été finalisé par l'auteur grâce à une subvention de la Régie régionale de Montréal-centre du ministère de la Santé et des Services sociaux. 
l'évaluateur s'est aussi intéressé à un troisième point de vue, soit celui des participants eux-mêmes, les « entraidants » pédophiles. D'où, finalement, une « triangulation " dans la démarche évaluative, telle que préconisée, par exemple, par Strupp et Hadley (1977), par Bednarz (1985), ou plus récemment en évaluation de programmes par Rossi et Freeman (1993).

\section{A. Sujets}

Le nombre de participants aux rencontres oscille généralement entre 10 et 14. Les 11 participants qui ont accepté de rencontrer l'évaluateur incluaient les deux plus anciens qui en étaient respectivement à leur $53^{\circ}$ et leur $56^{\mathrm{e}}$ présence mais aussi quatre nouveaux qui en étaient à leur début dans le groupe. En fait, la moyenne des participants en étaient à leur $18^{\mathrm{e}}$ présence $(\mathrm{ET}=19)$. On pouvait donc déceler, malgré une certaine mouvance, un noyau de plus anciens qui contribuait en quelque sorte à l'identité du groupe. Des 11 participants rencontrés, un seul n'avait plus de lien direct avec le système de santé ou le système judiciaire. Un autre était venu de lui-même au groupe mais gardait volontairement un lien direct avec un organisme extérieur. Initialement, les neuf autres participants avaient donc été orientés vers le programme par des agents externes, que ce soient des agents de probation $(n=3)$, des intervenants d'une prison ( $n=2)$, un intervenant d'une maison de transition $(n=1)$, un intervenant d'un CLSC $(n=1)$, le Centre de référence du Grand Montréal $(\mathbf{n}=1)$ et L'entraide pour hommes de Montréal $(\mathbf{n}=1)$.

Les liens de dépendance avec des organismes de référence peuvent amener à questionner le caractère plus ou moins volontaire de la participation de certains. Cet aspect a été abordé directement avec les animateurs, les agents externes et les participants. Effectivement, quelques participants semblaient vivre indirectement des pressions imposées par le système judiciaire, mais pas nécessairement pour qu'ils participent spécifiquement au programme Amorce. Il serait toutefois risqué de croire que ce contexte n'a eu aucune influence sur le témoignage, pourtant confidentiel, de certains participants devant l'évaluateur. Malgré cela, l'évaluateur a pu apprécier directement la grande franchise de plusieurs, notamment par rapport à des détails qu'ils n'avaient pas nécessairement à lui révéler.

L'âge des 11 participants variait de 25 à 71 ans $(M=46$ et $E T=14)$. La plupart occupaient un emploi (64\%), un seul étant retraité. Ils étaient tous considérés comme pédophiles dans l'esprit des animateurs, ce qui signifiait qu'ils avaient eu des contacts sexuels avec une ou plusieurs personnes mineures dans un contexte extrafamilial ou intrafamilial (inceste). Pour eux, le terme « pédophilie » était donc englobant et ignorait délibérément une certaine tendance qui voudrait que pères incestueux et véritables pédophiles soient deux sous-populations bien différentes, non seulement quant aux 
caractéristiques de leurs victimes mais aussi quant à toute leur dynamique particulière (Conte, 1990).

De toute façon, dans les faits, les données recueillies sur les 11 participants n'auraient probablement pas permis une distinction aussi claire entre ces deux sous-populations. En effet, six des participants pouvaient être considérés comme des "parents » incestueux (hétérosexuels) avec leur fille, leur belle-fille, leur nièce ou l'équivalent. Par contre, sept avaient agi hors de Ieur famille (avec des étrangers/ères). De ce dernier nombre, deux étaient pédophiles homosexuels et cinq pédophiles hétérosexuels. De plus, deux des participants, hêtérosexuels, avaient agi à la fois dans leur famille et à l'extérieur, d'où le recoupement de certaines données. Pour ce qui est de la judiciarisation, quatre des participants étaient en attente de condamnation ou de sentence ; sept étaient d'ex-détenus dont cinq étaient encore en période de transition; trois avaient été condamnés plus d'une fois pour des délits d'ordre sexuel et un l'avait déjà été pour des délits contre les biens.

L'évaluateur a aussi relevé que trois participants avaient déjà suivi une thérapie, que deux en poursuivaient toujours une, et que deux autres étaient en attente formelle d'admission. Ce demier chiffre est cependant trompeur puisque d'autres participants ont aussi mentionné qu'ils n'avaient pas les ressources financières pour demander une thérapie. Quoi qu'il en soit, il est bien certain qu'au moins sept des 11 participants avaient une expérience directe ou indirecte des services complémentaires de thérapie. C'est d'ailleurs le plus souvent dans ces termes que la majorité des participants décrivaient le rapport entre les deux formes d'aide et ils n'y voyaient pas d'incompatibilité.

\section{B. Instruments}

Quatre instruments ont été utilisés : trois questionnaires d'entretiens individuels semi-structurés (avec l'animateur, l'agent externe et le participant) et l' «Échelle de progression » de Chaffin (1992) inspirée de Finkelhor (1984). Par ailleurs, l'évaluateur avait signé avec chaque participant un document où en contrepartie de la permission d'étudier son dossier, il s'engageait à respecter la confidentialité des informations recueillies et son droit de retrait en tout temps.

L'Échelle de progression mesure, de manière plus quantitative que lors des entretiens semi-structurés, six éléments liés plus spécifiquement à la pédophilie : (1) perception de sa sexualité (congruence avec une sexualité normale), (2) attirance pour les enfants, (3) sexualité/affectivité avec des adultes, (4) contrôle de l'impulsivité, (5) responsabilisation et (6) empathie pour la victime. Chacun des items de l'échelle est gradué de 1 (inadaptation sévère) 
à 5 (exagération ou sur-contrôle, un score qui, finalement, n'a pas été attribué dans cette étude). La cote 4 représentait donc ici le critère de « réussite ». Au moment de la cotation de l'instrument, on indique également pour chaque item si la mesure représente une amélioration, une stabilisation, ou une détérioration par rapport à l'état antérieur. Aucun pré-test n'ayant pu être réalisé dans le contexte de cette évaluation de programme (par ailleurs très ponctuelle), il faut savoir que ce dernier aspect de la cotation correspond à une appréciation subjective du cheminement du participant depuis son arrivée dans le groupe. Par ailleurs, cette échelle de progression présume en quelque sorte que le participant traversera différents stades dans sa « carrière morale * d'abuseur (Goffman, 1961), notamment quant à l'étiquette sociale qu'il est prêt à porter (Collins, 1978).

\section{RÉSULTATS ET DISCUSSION}

Les données nécessaires à l'évaluation qualitative et quantitative du programme ont finalement pu être recueillies auprès des trois animateurs, de huit agents externes (l'un d'eux ayant référé deux clients) et de 11 participants.

\section{A. Services rendus}

Les rencontres de groupe, telles que l'évaluateur a pu les observer, se déroulent selon un ordre qui semble accepté de tous. Un des animateurs contrôle habituellement la durée de la rencontre et la séquence dans la prise de parole, alors que l'autre animateur (ou les deux autres) n'interviennent que sur des points précis. Le temps de discussion est donc surtout réservé à ce que les participants appellent eux-mêmes des « partages » ou des « témoignages ». Par ailleurs, chaque rencontre est précédée de deux minutes de silence utilisées pour la relaxation. Le plus souvent, les partages se rapportent à des difficultés vécues pendant la semaine ou bien à des prises de conscience : difficulté à éviter les situations à risque, frustration dans leurs contacts avec des intervenants extemes, problèmes d'intégration sociale, rejets familiaux, tracasseries juridiques, développements récents dans la relation amoureuse, craintes face au dévoilement à des membres de l'entourage, difficulté à reconnaître sa culpabilité, préoccupations sexuelles. Les discussions débouchent aussi parfois sur des thèmes plus universels, à savoir : la possibilité ou non de contrôler définitivement sa pédophilie, la spécificité de l'entraide et de la thérapie, la nécessité ou non d'avoir recours à des

4. Compte tenu du petit nombre de sujets, de certaines données manquantes et du peu de variabilité dans l'Échelle de progression de type Likert (ordinale), les résultats présentés plus loin ont plutôt servi d'indicateurs cliniques et les analyses statistiques ont été ignorées. 
professionnels, les ressources disponibles en communauté, la répétition du cycle agresseur/agressé, le poids du jugement social.

Aucun point de vue n'est alors imposé par les animateurs, même si certains participants plus anciens invoquent, quant à eux, leur propre expérience ou expertise. La consigne pour les participants ou les animateurs est de ne pas donner de conseils à un autre, surtout s'il n'en demande pas ouvertement. Pourtant, nous avons pu constater que le débat à ce propos n'est pas clos définitivement dans un tel groupe constamment en mutation. Le compromis actuel semble être qu'un participant peut raconter son propre vécu (surtout émotif) sur le sujet déjà évoqué par un autre, quitte à ce que ce dernier le prenne comme un conseil. Par ailleurs, il n'y a pas à Amorce de service de parrainage formalisé comme dans d'autres groupes d'entraide (Lavoie, 1989).

\section{B. Indices de satisfaction}

Les 11 participants ont exprimé leur satisfaction face au programme à $8,35$ en moyenne (ET $=0,8)$ sur une échelle graduée de nulle (1) à très élevée (10), ce qui reflète bien la nature positive de leurs commentaires généraux lors des entretiens individuels. Ils cotaient leur satisfaction envers les trois animateurs sensiblement au même niveau, soit à 8,5 en moyenne. Dans ce dernier cas, l'écart était cependant plus grand $(E T=1,6)$, ce qui correspond probablement à plus de personnalisation dans cette deuxième étape de la démarche. Dans la satisfaction qu'ils exprimaient par rapport au programme, les 11 participants insistaient surtout sur les effets bénéfiques du partage, de l'entraide et du dévoilement. De constater qu'ils n'étaient pas les seuls dans leur situation d'hommes pédophiles leur permettait de briser leur isolement, alors que le fait d'en rencontrer qui avaient cheminé plus ou moins qu'eux leur permettait de se donner du courage et de mieux se positionner. Quoique ce positionnement qu'ils disaient avoir opéré pût parfois être discutable (d'un point de vue externe), il n'en restait pas moins qu'il semblait meilleur que la confusion qui avait été la leur auparavant.

En effet, les participants ont souvent indiqué à l'évaluateur qu'ils avaient vếcu des périodes où ils ne réagissaient même plus à ce qui leur arrivait. Certes, ces périodes correspondaient souvent à des étapes difficiles du processus judiciaire où ils n'étaient pas toujours très motivés ou disposés à accepter qu'il fallait changer. N'empêche qu'ils vivaient alors dans l'immobilisme et qu'ils ne pouvaient rien entreprendre (de positif ou de négatif). «Auparavant, je ne voulais que rester couché et ne plus rien faire ", nous rapportait ainsi l'un des participants. Un autre ajoutait : « Le groupe n'a pas réglé mon problème de pédophilie. Il m'a plutôt aidé à placer les choses... quand j'étais mêlé. » Les témoignages n'ont pas tous été aussi 
convaincants, mais il n'en reste pas moins que les situations de stabilisation, de clarification, de mise en perspective, de stimulation au changement, étaient rapportées par la majorité des participants. Quoique cela puisse sembler bien minime à certains, ce n'en est pas moins bien spécifique à la problématique centrale des hommes pédophiles. D'une certaine façon, il s'agit là, en effet, de la condition première à tout changement ultérieur en thérapie ou autrement. Or, selon ce qu'en disaient certains agents externes au programme, l'entraide pourrait justement être plus efficace que les thérapies à ce stade-ci du cheminement d'un homme pédophile.

Quant aux différents agents externes contactés individuellement, ils s'estimaient généralement satisfaits de leur coopération au programme, sinon de l'évolution de leurs propres clients dans ce programme. Même au chapitre de la confidentialité à respecter ou de l'échange mutuel d'information avec les animateurs, ils se montraient également respectueux des règles du groupe : " Ca leur appartient ce qu'ils disent là-bas », ou bien «On respecte qu'il se fasse des choses en parallèle, sous un autre angle ». Par-delà ce qu'ils auraient aimé savoir, malgré tout, sur le contenu des discussions de groupe, la plupart des agents externes se sentaient donc rassurés de savoir que leurs clients avaient un lieu où cheminer quant à leur pédophilie (surtout pour tous ceux qui n'étaient pas en thérapie). En effet, ils admettaient eux-mêmes leurs limites à ce niveau et ils s'en remettaient, en fin de compte, à la satisfaction exprimée ouvertement par leurs clients en regard du programme.

\section{Niveaux atteints dans le cheminement des participants}

Quant aux six caractéristiques mesurées à l'Échelle de progression, le tableau 1 nous indique à quelle étape se situaient, en moyenne, les 11 participants au moment de l'évaluation, et ce, selon le point de vue des animateurs, des agents extemes et des participants eux-mêmes. Cette évaluation statique ne présume pas cependant des progrès qui avaient pu être réalisés depuis leur début au programme (lesquels seront analysés plus loin). Ces résultats nous indiquent que, selon les animateurs, la moyenne des participants se situerait assez près d'une certaine « normalité » (i.e. près du niveau 4) pour ce qui est des deux derniers éléments, soit « responsabilisation » et " empathie pour la victime ». Ce seraient là des thèmes qui, spontanément, sont abordés plus fréquemment lors des rencontres du groupe d'entraide, d'ou une certaine validation de ces résultats particuliers. Par contre, et ceci toujours selon les animateurs, les participants auraient plus de difficulté pour ce qui est de la « perception de leur sexualité » et de leur « sexualité/affectivité avec des adultes ». Effectivement, il s'agit de thèmes qui, bien qu'ils préoccupent les participants, ne sont abordés qu'avec difficulté en groupe. 
Tableau 1

Niveaux atteints chez les participants, selon les animateurs, les agents externes et les participants

\begin{tabular}{lcccc}
\hline Elément d'évaluation & Animateurs & $\begin{array}{c}\text { Agents } \\
\text { externes } \\
(\mathrm{n}=8)\end{array}$ & Participants & $\begin{array}{c}\text { Niveau } \\
\text { moyen }\end{array}$ \\
\hline 1. Perception/sexualité & $(\mathrm{n}=3)$ & 2,83 & 2,80 & $\mathbf{2 , 9 3}$ \\
2. Attirance/enfants & 3,17 & 2,71 & 3,46 & $\mathbf{3 , 2 1}$ \\
3. Avec des adultes & 3,46 & 2,17 & 2,90 & $\mathbf{2 , 7 2}$ \\
4. Impulsivité & 3,09 & 3,40 & 3,55 & $\mathbf{3 , 4 3}$ \\
5. Responsabilisation & 3,35 & 3,13 & 3,09 & $\mathbf{3 , 3 6}$ \\
6. Empathie/victime & 3,87 & 2,71 & 3,09 & $\mathbf{3 , 2 4}$ \\
\hline \multicolumn{1}{c}{ Niveau moyen } & 3,90 & 2,83 & 3,15 & $\mathbf{3 , 1 5}$ \\
\hline
\end{tabular}

Quant aux agents externes qui suivaient encore les participants, ils ont produit des estimations qui avaient le mérite d'apporter un point de vue différent de celui des animateurs. En effet, la deuxième colonne du tableau 1 nous indique que, selon eux, les participants se situaient, en moyenne, à des niveaux plus bas $(2,83)$ par rapport à ce qu'estimaient les animateurs $(3,47)$. Cette différence entre les points de vue des animateurs et des agents externes n'apparaît cependant pas du tout à l'élément «impulsivité ». Nous devons donc postuler ici que les animateurs, en plus d'être soumis à leurs propres attentes de réussite, ont une connaissance des participants qui est bien différente de celle des agents externes. Il faut voir, en effet, que les animateurs ne sont évidemment pas en situation de contrôle face aux participants et qu'ils ne les confrontent pas, surtout par rapport à leur quotidien qu'ils ignorent d'ailleurs. Quant aux agents externes qui, eux, n'ont pas accès au contenu des discussions de groupe, ils ont le rôle ingrat de relever constamment les fautes de leurs clients, alors qu'ils servent d'intermédiaires entre eux et la société. En fait, ces agents externes étaient effectivement soit des agents de probation, soit des intervenants de maison de transition, un seul étant intervenant dans une thérapie et un autre étant le propre avocat du participant. Les opinions étaient donc très diversifiées chez ces personnes, ce qui se reflétait d'ailleurs par des différences plus larges dans les cotations aux divers éléments, les écarts type variant de 0,8 à 1,4.

Quant à la moyenne des participants eux-mêmes, ils se sont positionnés exactement à mi-chemin entre l'opinion des animateurs $(3,47)$ et celle, plus négative, des agents externes $(2,83)$. Plus spécifiquement, l'évaluation des participants était plus proche de celle des agents externes pour les éléments 1, 5 et même 6. Par contre, elle était plus proche de celle des animateurs pour ce qui est des éléments 2 et 3 . Quant à l'élément 4 (« impulsivité »), il était estimé au même niveau moyen selon les trois points de vue. 
Finalement, la dernière colonne du tableau 1 nous permet de dégager l'essentiel des trois points de vue exprimés précédemment : c'est bien par rapport à la « perception de leur sexualité » et à la « sexualité/affectivité avec des adultes » que les participants ont encore le plus de difficulté.

\section{Effets spécifiques à la pédophilie}

L'Échelle de progression permettait aussi d'estimer jusqu'à quel point le participant avait progressé depuis son entrée au programme, et ce, quant aux six éléments spécifiques à la pédophilie. Encore ici, le devis d'évaluation permettait de prendre en compte l'appréciation des animateurs, des agents externes et des participants eux-mêmes. Par contre, ce type de devis non expérimental ne permettait pas vraiment, il faut bien s'en rappeler, de déterminer si les changements observés étaient dus au programme d'entraide ou à des facteurs parallèles : thérapie déjà en cours, passage du temps, problèmes concurrents résolus... Pour ce qui est des « améliorations » que les 11 participants auraient démontrées depuis le début de leur engagement au programme, le tableau 2 nous indique donc que, selon les animateurs, de 64 à $77 \%(M=69 \%)$ d'entre eux se seraient améliorés, dépendamment de l'indicateur qui est considéré. Il faut se rappeler ici que plusieurs participants étaient relativement nouveaux au programme, ce qui a parfois amené les animateurs à ne pas vraiment coter une évolution sur certains éléments. Dans ce cas, ils étaient alors cotés " stables », aucun n'ayant d'ailleurs « régressé ». Dans l'ensemble, les participants auraient donc surtout fait des progrès par rapport à l'élément " perception de leur sexualité », les progrès étant à peu près égaux sur tous les autres éléments.

Tableau 2

Pourcentages de ceux qui ont progressé, selon les animateurs, les agents externes et les participants

\begin{tabular}{lcccc}
\hline Élément d'évaluation & Animateurs & $\begin{array}{c}\text { Agents } \\
\text { externes } \\
(\mathbf{n}=\mathbf{8})\end{array}$ & $\begin{array}{c}\text { Participants } \\
(\mathrm{n}=11)\end{array}$ & $\begin{array}{c}\text { Niveau } \\
\text { moyen }\end{array}$ \\
\hline 1. Perception/sexuaité & 77 & 40 & 50 & $\mathbf{5 6}$ \\
2. Attirance/enfants & 64 & 0 & 9 & $\mathbf{2 4}$ \\
3. Avec des adultes & 67 & 40 & 50 & $\mathbf{5 2}$ \\
4. Impulsivité & 65 & 0 & 18 & $\mathbf{2 8}$ \\
5. Responsabilisation & 69 & 25 & 9 & $\mathbf{3 4}$ \\
6. Empathie/victime & 72 & 14 & 9 & $\mathbf{3 2}$ \\
\hline \multicolumn{1}{c}{ Pourcentage moyen } & 69 & 20 & 24 & $\mathbf{3 8}$ \\
\hline
\end{tabular}


Selon les agents externes, ce ne seraient toutefois que $20 \%$ des participants, pour l'ensemble des six éléments, qui auraient progressé depuis leurs débuts au programme, ce qui est beaucoup plus bas que l'estimation des animateurs. Ici, compte tenu des données manquantes, le petit nombre des observations aurait pu cependant fausser l'estimation. En effet, certains éléments particuliers n'étaient pas cotés par des agents externes qui s'estimaient souvent mal informés quant à ceux-ci. Néanmoins, l'évaluation globale des agents externes était certainement moins optimiste, quoique relativement bonne (pour une clientèle aussi vulnérable) aux éléments 1 et 3 (40\% dans les deux cas). Par ailleurs, ces deux scores moyens se rapprochaient de ceux déjà notés par le plus expérimenté des trois animateurs (soit $50 \%$ en moyenne). Quant au fait que les agents externes n'aient noté aucune amélioration aux éléments 2 et 4 , cela pourrait correspondre, en partie, à beaucoup plus de craintes quant à des facteurs dont, souvent, ils ont personnellement la responsabilité d'assurer le contrôle. Pour l'ensemble de leurs estimations, il faut savoir aussi qu'il leur arrivait parfois de ne pas noter de progrès parce qu'ils estimaient que les progrès étaient déjà accomplis « avant » l'entrée au programme d'entraide. Par ailleurs, notons que lors de l'entretien téléphonique, la majorité des agents externes estimaient que leurs clients avaient progressé pendant leur participation au programme mais pas nécessairement par rapport aux conditions suggérées dans l'Échelle de progression. Par exemple, ils mentionnaient plus d'ouverture, plus de socialisation, plus de prise en charge, plus de participation à des activités structurantes et plus d'empathie face aux coparticipants (mais pas nécessairement face à leurs victimes).

La moyenne des estimations des 11 participants (sur leurs propres progrès) était finalement assez semblable à celle des agents externes, ce qui démontre probablement beaucoup de modestie ou de réserve chez eux. Également, tout comme les agents externes, ils identifiaient eux aussi deux éléments de progrès comme déterminants : "perception de leur sexualité » et « sexualité/affectivité avec des adultes », le premier étant d'ailleurs déjà bien noté par les animateurs.

Finalement, la dernière colonne du tableau 2 nous confirme surtout le point de vue des agents externes et des participants quant à l'importance des progrès réalisés aux éléments 1 et 3 . Faut-il croire que les autres éléments sont plus statiques ou bien déjà fixés avant le début au programme ? Faut-il croire que les autres éléments avaient déjà été pris en charge en thérapie (dans le cas de certains participants) ou lors des rencontres avec les agents extemes? Par ailleurs, il faut se rappeler que, au tableau 1 (niveaux atteints), c'était sur ces mêmes deux éléments que les participants étaient cotés au plus bas. Il faudrait donc conclure, de façon un peu paradoxale, que c'est là où les participants progressent le plus mais que c'est aussi là qu'il y a encore beaucoup de cheminement à faire. 
Malgré la petitesse de l'échantillon de participants, les progrès réalisés aux éléments 1 et 3 nous amèneraient aussi à formuler l'hypothèse suivante : la dynamique d'un groupe d'entraide permettrait aux hommes pédophiles de progresser davantage par rapport à leur sexualité avec des adultes (sexualité de remplacement) que par rapport à leur sexualité avec des enfants (sexualité déviante). À l'inverse, Chaffin (1992) lui-même avait noté que, dans le cadre cette fois d'une thérapie structurée, c'était sur ces deux éléments que les progrès étaient les plus faibles. En somme, les groupes d'entraide pourraient donc être particulièrement efficaces à combler le vide créé par la répression de la sexualité avec des enfants. Il s'agirait donc là d'une hypothèse à vérifier, mais cette fois dans un contexte de recherche plutôt que d'évaluation de programmes.

Quant à l'ampleur réelle des résultats globaux obtenus par les participants au programme (moyenne globale des pourcentages au tableau 2), elle doit être estimée à sa juste valeur. Tout d'abord, le fait que seulement $38 \%$ des participants, en moyenne, aient progressé cache tout de même une réalité plus optimiste. En fait, si nous considérions plutôt qu'un participant s'est amélioré dès qu'il a marqué un progrès à l'un ou l'autre des six éléments, ce serait alors $68 \%$ des participants qu'il faudrait considérer ici. Il s'agirait là d'une moyenne entre le point de vue des animateurs $(87 \%)$, des agents externes $(63 \%)$ et des participants $(55 \%)$, lesquels points de vue seraient d'ailleurs beaucoup moins éloignés selon cette méthode de compilation. Quoi qu'il en soit au sujet de la façon de calculer un taux de réussite, il n'en demeure pas moins que même un taux de $38 \%$ serait déjà appréciable, compte tenu notamment qu'il s'agissait ici d'hommes pédophiles qui n'en étaient souvent qu'à quelques rencontres au programme Amorce. Idéalement, la durée d'exposition aux bienfaits éventuels d'un tel programme devrait effectivement être beaucoup plus longue (Roundy et Horton, 1988). Un point de vue qui est partiellement confirmé du fait que le nombre d'éléments d'évaluation (sur l'Échelle de progression) où les participants avaient progressé était en relation (faible mais positive) avec le nombre de rencontres auxquelles ils avaient assisté $(r=0,42)$.

\section{E. Effets plus larges}

Les entretiens semi-structurés ont aussi mis à jour des effets bénéfiques du programme qui ne semblaient pas, de prime abord, directement liés à la problématique de la pédophilie (telle que mesurée du moins par l'Échelle de progression). Pourtant, lorsqu'on aborde le problème de façon systémique et que l'on connaît l'importance des facteurs concomitants dans la commission des délits, on ne peut s'empêcher d'accorder de l'intérêt à ces aspects plus larges. Certains participants, notamment ceux ayant déjà participé à des 
thérapies pour hommes pédophiles, insistaient d' ailleurs eux-mêmes sur l'importance de ces facteurs qui sont plus ou moins associés à la pédophilie ou au passage à l'acte. Cette approche plus qualitative de l'évaluation du programme Amorce a donc révélé que :

- La majorité des participants vivaient moins de solitude depuis leur adhésion au groupe. Ils ne se fréquentaient pas en dehors des rencontres mais ils avaient néanmoins l'impression qu'ils n'étaient plus seuls avec leur problème de pédophilie. Le fait de savoir qu'ils pouvaient également communiquer, au besoin, avec un animateur du programme semblait également rassurant, même si, en pratique, peu utilisaient ce service.

- Un participant témoignait qu'il avait mieux traversé un deuil récent grâce au soutien reçu dans le groupe. Encore là, nous pouvons croire qu'il a appris ainsi à demander de l'aide et, possiblement, à éviter des périodes de rechute.

- Un participant décrivait dans quel état il était à sa sortie de prison. Il se laissait aller, ne voulait que dormir, n'entreprenait plus rien, n'avait plus d'espoir. Il est sorti de cet état (qui, rétrospectivement, évoque la dépression) très rapidement à la suite de ses premières rencontres avec le groupe d'entraide. L'intervenant qui le suit encore aujourd'hui a confirmé cette version et affirme avoir été surpris de la rapidité du changement observé.

- Plusieurs participants ont mentionné qu'ils étaient devenus sensibles à la souffrance d'autrui en écoutant les témoignages d'entraide. L'empathie pour d'autres êtres humains, ceci peut-être par une dynamique d'identification, semblait un phénomène nouveau chez plusieurs. Les participants ne faisaient toutefois pas nécessairement un lien direct entre l'empathie pour d'autres pédophiles agresseurs et l'empathie pour les victimes de leurs gestes, ce qui pourrait sembler, de prime abord, bien injuste pour les victimes. Pourtant, les résultats décrits plus hauts à l'Échelle de progression suggèrent justement (du moins d'après les animateurs) que le groupe d'entraide a un certain effet bénéfique quant à l'empathie pour la victime. Il faudrait donc croire ici que pour réussir à avoir de l'empathie pour la victime, il faut d'abord être capable d'empathie pour ses semblables, ce qui, en soi, mériterait d'être approfondi dans un autre contexte de travail. Par ailleurs, certains participants rappelaient qu'eux-mêmes avaient déjà été abusés sexuellement dans leur enfance et donc qu'ils avaient aussi été des victimes. Ils avaient ainsi conscience de répéter un cycle abuseur-abusé, ce qui n'était néanmoins pas toujours évident lorsqu'ils étaient confrontés dans l'immédiat aux accusations de leurs propres victimes.

- Un participant mentionnait aussi que les autres membres l'avaient motivé à revenir sur le marché du travail. 
- À sa première rencontre, un participant était incapable de s'exprimer devant les autres. Par la suite, il remerciait cependant avec émotion les autres participants en expliquant qu'il avait été touché, qu'il était désormais moins seul, et que très bientôt il serait capable de prendre la parole.

- Un participant rappelait aussi qu'il était maintenant prêt pour entreprendre une vie amoureuse avec une personne adulte, ce qui n'avait pas nécessairement un rapport direct avec le contrôle de sa pédophilie qui semblait assuré. Il s'agissait pourtant alors de combler le vide créé justement par ce contrôle de la pédophilie, ce en quoi le groupe d'entraide l'aurait beaucoup aidé.

- L'atmosphère créée par les témoignages individuels dans le groupe serait propice à créer, chez certains, ce qu'un intervenant externe a appelé une « décharge émotive », alors que cet effet est particulièrement recherché chez ceux qui sont longtemps restés repliés sur eux-mêmes. S'en est suivie, selon cet intervenant, une suite de démarches positives.

- Certains participants expliquaient que le groupe d'entraide leur servait à régler des problèmes plus immédiats alors que la thérapie les aidait pour des problèmes à plus long terme.

- Quant à la responsabilisation plus large des individus, il nous a semblé qu'elle ne pouvait s'amorcer qu'à partir d'un positionnement adulte dans la société. Or, les participants au programme Amorce ont mentionné qu'ils appréciaient justement d'être considérés ici comme des adultes responsables plutôt que comme des clients.

- Certains notaient avoir traversé des périodes de dépression où ils ne semblaient pas pouvoir trouver d'aide hors du groupe. L'un d'eux mentionnait même avec humour « avoir été ramassé à la petite cuillère " à une certaine époque. D'ailleurs, l'évaluateur lui-même a pu constater que certains participants étaient encore très perturbés lors des rencontres, ce qui semblait les inciter à demeurer dans le groupe.

- Un nouveau participant ne croyait pas avoir fait de progrès jusqu'à présent mais il écoutait attentivement les témoignages positifs des autres, ce qui lui donnait de l'espoir : « Un jour, dans un partage, moi aussi je vais comprendre. »

- Certains participants avouaient vivre de grandes pressions dans l'immédiat. Pour eux, le fait de venir au groupe ne réglait pas nécessairement leurs problèmes mais permettait tout de même un moment de relâche. Dans ce cas, le groupe semblait agir comme « contenant » pour des « contenus » (émotifs, notamment) qu'ils n’étaient pas aptes à gérer seuls. 
- Pour un participant, le groupe avait permis d'améliorer ses habiletés de communication, ce qui avait amélioré ses rapports avec sa conjointe.

- Pour certains participants, le groupe d'entraide permettait de regagner une certaine dignité et de sortir de la stigmatisation imposée par la société.

Finalement, les effets observés ici correspondraient d'une certaine façon à ce que Lavoie (1989) identifie comme les « facteurs aidants » de l'entraide : « le soutien émotif, la rencontre de semblables, la révélation de soi, la clarification d'un problème et l'information " (p. 82-87), bien que ce soit à un moindre niveau pour le dernier facteur. Certains agents externes, eux, conceptualisaient plutôt les changements survenus grâce à l'entraide en termes de « conscientisation » ou bien de dilution de la « négation » et de la « minimisation ». Pour eux, comme c'est d'ailleurs l'opinion dans le milieu thérapeutique (Barbaree, 1991), il s'agirait là d'un préalable à réaliser chez le délinquant avant tout autre progrès, et l'entraide y contribuerait de façon particulièrement efficace.

\section{CONCLUSION}

Pour réaliser sa tâche, l'évaluateur de ce programme a analysé la documentation pertinente et rencontré ou contacté individuellement les participants, les animateurs, le responsable de l'administration, les membres du Comité aviseur et divers représentants d'organismes extérieurs. Les rencontres du groupe d'entraide ont également été observées. L'évaluateur a, entre autres méthodes, appliqué un instrument de mesure déjà éprouvé, mais il s'est surtout inspiré d'une approche plus qualitative lors d'entretiens semistructurés. Il a donc cherché à rester disponible à ce qui émergeait d'un programme qui, de prime abord, n'était pas conventionnel. C'est ainsi qu'il a surtout orienté sa démarche en fonction des principes et des besoins propres à un groupe d'entraide plutôt qu'à une thérapie spécialisée.

Le but premier de cette évaluation d'un programme d'entraide n'était pas non plus d'en déterminer formellement l'efficacité, laquelle ne serait d'ailleurs pas facilement perceptible ici parmi d'autres facteurs contribuant au changement (Powell, 1987). Il n'en reste pas moins que, en pondérant trois points de vue différents, on observerait ici que l'approche d'entraide est particulièrement efficace chez 52 à $56 \%$ des hommes pédophiles selon que I'on parle de "sexualité/affectivité avec des adultes" ou bien de "perception de leur sexualité ». Si nous considérons leurs progrès quant à l'une ou l'autre des caractéristiques pédophiliques ciblées pour l'évaluation, ce seraient même $68 \%$ de ceux-ci qui se seraient améliorés après une moyenne de 18 rencontres. De plus, considérant qu'il ne s'agissait absolument pas ici d'une thérapie où ces problèmes auraient été traités systématiquement, 
nous pourrions alors conclure à des résultats fort appréciables dans le contexte (Patton, 1991).

Une approche plus qualitative de la question a même révélé un effet encore plus grand, si l'on retient des critères moins spécifiques à la pédophilie. En effet, la majorité des participants témoignaient des effets bénéfiques du programme, que ce soit au niveau de la rupture de l'isolement, de la modification des attitudes, de l'augmentation de l'empathie, de la motivation à changer, des habiletés de communication, de la « décharge émotive ", de la clarification des problèmes immédiats, d'un repositionnement social adulte, du soutien lors des phases dépressives ou d'une atténuation de la tension.

Finalement, la présente évaluation du programme aura surtout permis, par-delà une stricte évaluation de son efficacité, une analyse de son processus de fonctionnement. De cette analyse plus globale émergent certaines forces, déjà partiellement soulignées dans les sections précédentes, mais aussi, cette fois, des faiblesses (tableau 3 ). Nous y voyons que certaines forces découlent justement de l'application des principes de l'entraide ou de l'approche communautaire. De la même façon, certaines faiblesses pourraient être reliées aussi à des modes de fonctionnement typiques du milieu (par exemple, au niveau du soutien administratif). Le tableau d'ensemble reste toutefois

\section{Tableat 3}

Forces et faiblesses du programme

\section{FORCES}

- Effets spécifiques à la pédophilie

- Effets plus larges sur des problèmes connexes

- Dynamique propre a l'entraide et au partage

- Motivation et disponibilité : D'un noyau de participants convaincus

Des trois animateurs

Du personnel de l'organisme d'entraide

D'un réseau de soutien externe

- Ouverture à la critique : $\quad$ Cornité aviseur d'experts

Recours à l'évaluation de programme

- Caractère novateur, unique et progressif du programme

- Approche communautaire et non médicale du problème

- Approche préventive

- Respect et responsabilisation des participants

\section{FAIBLESSES}

- Positionnement à améliorer dans le réseau de la santé et de la justice

- Promotion à élargir vers d'autres ressources externes

- Sensibilisation sociale à améliorer quant au problème de la pédophilie

- Soutien administratif à développer

- Recentration plus poussée des thèmes de discussion sur la sexualité 
relativement favorable au programme Amorce, d'autant plus que certaines recommandations relatives aux faiblesses observées étaient déjà en voie d'application.

Dans l'ensemble, il faut cependant reconnaître aussi que les résultats présentés ici doivent être interprétés avec prudence, notamment ceux concernant les participants eux-mêmes. En effet, le faible échantillon qui a été analysé limite les possibilités de généralisation alors que ces participants, de par leur condition ou en raison d'un effet de demande, ont pu apporter à l'évaluateur des témoignages biaisés. De même, les résultats quant aux progrès qu'auraient réalisés ces participants proviennent d'une interprétation subjective qui n'a pas la rigueur d'une procédure test-retest. Néanmoins, une telle évaluation de programme nous apporte suffisamment d'information pour confirmer la valeur de l'expérience qui est actuellement en cours dans un domaine qui a justement besoin d'innovation.

\section{BIBLIOGRAPHIE}

BARBAREE, H. E. (1991), « Déni de la réalité et minimisation par les délinquants sexuels : évaluation et résultats du traitement ", Forum. Recherche sur l'actualité correctionnelle, vol. $3, \mathrm{n}^{\circ} 4$, p. $35-38$.

BEDNARZ, D. (1985), "Quantity and quality in evaluation research : A divergent view », Evaluation and Program Planning, vol. 8, p. 289-306.

CHAFFIN, M. (1992), « Factors associated with treatment completion and progress among intrafamilial sexual abusers , Child Abuse and Neglect, vol. 16, p. 251-264.

COLLINS, M. C. (1978), Child abuser. A study of child abusers in self-help group therapy, Littleton, Mass., PSG Publishing.

CONTE, J. R. (1990), «The incest offender : An overview and introduction », in A. L. HORTON, B. L. JOHNSON, L. M. ROUNDY et D. WILLIAMS (dir), The incest perpetrator (p. 19-28), Newbury Park, Sage.

FINKELLHOR, D. (1984), Child sexual abuse : New theory and research, New York, The Free Press.

GIARRETTO, H. (1982), Integrated treatment of child sexual abuse : A treatment and training manual, Palo Alto, Calif., Science and Behavior Books.

GIARRETTO, H. et EINFELD-GIARRETTO, A. (1990), « Integrated treatment : The selfhelp factor ", in A. L. HORTON, B. L. JOHNSON, L. M. ROUNDY et D. WILLIAMS (dir), The incest perpetrator (p. 219-226), Newbury Park, Sage.

GOFFMAN, E. (1961), Asylums: Essays on the social situations of mental patients and other inmates, Garden City, NJ, Anchor Books.

HEDRICK, H. L., ISENBERG, D. H. et MARTINI, C. J. M. (1992), « Self-help groups : empowernent through policy and partnerships ", in A. H. KATZ, H. L. HEDRICK, D. H. ISENBERG, L. M. THOMPSON, T. GOODRICH et A. H. KUTSCHER (dir), Self-help. Concepts and applications (p. 3-55), Philadelphia, Charles Press.

HERMAN, J. L. (1981), Father-daughter incest, Cambridge, Mass., Harvard University Press. 
KRAMER, K. D. et NASH, K. B. (1992), "Self-help group models : An ecological conceptualization », in A. H. KATZ, H. L. HEDRICK, D. H. ISENBERG, L. M. THOMPSON, T. GOODRICH et A. H. KUTSCHER (dir), Self-help. Concepts and applications (p. 144-148), Philadelphia, Charles Press.

LAVOIE, F. (1989), « L'évaluation des groupes d'entraide ", in J. M. ROMEDER (dir), Les groupes d'entraide et la santé. Nouvelles solidarités (p. 77-98), Ottawa/Montréal, Conseil canadien de developpement social.

PATTON, M. Q. (1991), « Patterns, themes, and lessons ", in M. Q. PATTON (dir.), Family sexual abuse. Frontline research and evaluation (p. 223-242), Newbury Park, Sage.

PELLERIN, B., PROULX, J., OUIMET, M., PARADIS, Y, MCKIBBEN, A. et AUBUT, J. (1996), « Étude de la récidive post-traitement chez des agresseurs sexuels judiciarisés », Criminologie, vol. 29, n' 1, p. 85-108.

POWELL, T. J. (1987), Self-help organizations and professional practice, Silver Spring, Maryland, National Association of Social Workers.

ROMEDER, J. M. (dir.) (1989), Les groupes d'entraide et la santé. Nouvelles solidarités, Ottawa/Montreal, Conseil canadien de développement social

ROSSI, P. H. et FREEMAN, H. E. (1993), Evaluation. A systematic approach, Beverly Hills, Sage.

ROUNDY, L. M. et HORTON, A. L. (1988), « Professional and treatment issues for clinicians who intervene with incest perpetrators $"$, in A. L. HORTON, B. L. JOHNSON, L. M. ROUNDY et D. WILLIAMS (dir.), The incest perpetrator. A family member no one wants to treat (p. 164-189), Newbury Park, Sage.

SILVERMAN, P. R. (1992), « Critical aspects of the mutual help experience ", in A. H. Katz, H. L. Hedrick, D. H. Isenberg, L. M. THOMPSON, T. GOODRICH et A. H. KUTSCHER (dir.), Self-help. Concepts and applications (p. 76-89), Philadelphia, Charles Press.

STRUPP, H. H. et HADLEY, S. W. (1977), «A tripartite model of mental health and therapeutic outcomes \#, American Psychologist, vol. 32, p. 187-196.

VÉRIFICATEUR GÉNÉRAL DU CANADA (1996), Rapport sur le Service correctionnel du Canada, chapitre 10, Ottawa.

WILSON, R. J. (1996), « Une situation sans issue : jusqu'où les psychologues peuvent-ils aller pour aider les délinquants après l'expiration de leur peine ?", Forum. Recherche sur l'actualité correctionnelle, vol. 8, $\mathrm{n}^{\circ} 3$, p. 27-29. 